\title{
Hepatitis B surface antigen-negative, but HBV DNA-positive patients in Bangladesh
}

\author{
Mahtab MA ${ }^{1}$, Akbar $\mathrm{SMF}^{2}$, Rahman $\mathrm{S}^{1}$ \\ ${ }^{1}$ Department of Hepatology, Bangabandhu Sheikh Mujib Medical University, Dhaka, Bangladesh, \\ ${ }^{2}$ Department of Medical Sciences, Toshiba General Hospital, Tokyo, Japan. \\ Emial: shwapnil@agni.com
}

\begin{abstract}
Hepatitis B surface antigen (HBsAg) is regarded as sole marker of hepatitis B virus (HBV) infection in Bangladesh and most other developing countries. However, some HBV-negative subjects may harbor HBV DNA and transfusion of their blood may cause HBV infection in recipients. HBV DNA was checked in 20 patients with cryptogenic liver cirrhosis, 10 patients with hepatocellular carcinoma without any known etiology, and 10 apparently healthy subjects with elevated levels of serum alanine aminotransferase (ALT). HBV DNA was detected in 8 of 20 patients with cryptogenic liver cirrhosis, 1 of 10 patients with hepatocellular carcinoma, and 2 of 10 apparent healthy subjects with elevated ALT. However, all of them were negative for HBsAg in the sera. This study indicates that some additional mechanisms should be developed for detection of $\mathrm{HBsAg}$-negative $\mathrm{HBV}$-infected subjects for efficient control and management of HBV infection in Bangladesh.
\end{abstract}

\section{Introduction}

Hepatitis B virus (HBV) is a major cause of liver disease worldwide. The development and use of a vaccine for HBV along with blocking of vertical and horizontal transmission of HBV has resulted in substantial decline in the numbers of new cases of HBV infection among children, adolescents and adults in developed countries ${ }^{1}$. However, this success has not been duplicated worldwide. Both acute and chronic HBV infections continue to represent important health problems in most developing countries ${ }^{2}$. It is true that socioeconomical factors and poorly-developed health care delivery system mainly contribute to ineffective control of HBV in developing countries. However, recent studies have provided new and novel information about $\mathrm{HBV}$ virology that should be incorporated for designing $\mathrm{HBV}$ control and management strategies ${ }^{3}$.

Presence of HBV DNA in the liver represents the gold standard of HBV infection. For practical purposes, however, hepatitis B surface antigen (HBsAg) is estimated in the sera to assess if some one is infected with the HBV or not. Assessment of HBsAg is comparatively cheaper and this can be accomplished in most, if not all, medical facilities of developing countries. On the other hand, HBV DNA can be assessed in only few sophisticated laboratories of developing countries and most of these centers are located in big cities. Also, estimation of HBV DNA is costly and most of the developing countries are not endowed with statesupported health insurance system. If some one is negative for HBsAg in the sera, it is concluded that he or she is free from HBV infection. However, it is now evident that a group of $\mathrm{HBV}$-infected persons may not express HBsAg in the sera due to various factors $^{4-9}$. Increased replication of $\mathrm{HBV}$ DNA with flare of ALT and severe liver damages has been reported in these patients either spontaneously or due to other pathological conditions or for using immune suppressive drugs $^{\mathbf{1 0 - 1 2}}$. In addition, HBsAg-negative subjects are allowed to donate blood and other body fluids in most developing countries without assessment of HBV DNA in their sera.

Bangladesh belongs to the intermediate prevalence region for $\mathrm{HBV}$ infection, and studies have shown that $\mathrm{HBV}$ is the major etiological agent of acute hepatitis, chronic hepatitis, cirrhosis of liver and hepatocellular carcinoma in Bangladesh ${ }^{13}$. Several cases of post-transfusion hepatitis have occurred in our hospitals after transfusion of HBsAg-negative blood (personal communication), however, nothing is known about the entity of occult HBV infection or $\mathrm{HBsAg}$-negative $\mathrm{HBV}$-infected subjects in Bangladesh.

This study was done to provide direct evidence about existence of occult HBV infection in 
Bangladesh. We encountered a group of patients with cryptogenic liver diseases that were negative for serological markers of hepatitis A, B, C, and E viruses. Also, some apparently-healthy individuals had persistently increased ALT values above upper limit of normal (ULN, >42 U/L) without any subjective symptoms of liver diseases. Serum HBV DNA was checked in these subjects to provide insights about entity of HBsAg-negative HBVinfected subjects in Bangladesh.

\section{Materials and Methods}

Patients: The study was conducted from January 2010 to December 2011. The study was conducted at Department of Hepatology, Bangabandhu Sheikh Mujib Medical University (BSMMU) and LabAid Specialized Hospital (LSH), Dhaka where patients with liver cirrhosis and HCC have been attending our clinics for regular follow up and treatment. Patients fulfilling the inclusion criteria and consenting to be part of the study were included. Since occult hepatitis B is an uncommon entity, sample size determination was not possible. Only the first 20 patients with cryptogenic liver cirrhosis, 10 patents with hepatocellular carcinoma (HCC) of unknown etiologies, and 10 apparently health subjects with elevated levels of ALT in the sera were enrolled in this study. Informed written consent was obtained from all patients and the study was approved by the appropriate ethics committee of Bangabandhu Sheikh Mujib Medical University (BSMMU). All patients were negative for IgM type antibody to HAV, anti-HCV, and antiHEV IgM in the sera. Also, HBsAg were not detected in any of these patients. None of them had features of autoimmune hepatitis and they had no history of alcohol abuse (usually people of Bangladesh do not use alcohol due to religious factors). Serological markers of HBV \& HCV were checked at least 2 times before a diagnosis of cryptogenic liver diseases was made in patients with liver cirrhosis and HCC. Subjects with elevated ALT were not obese and a diagnosis of fatty liver was discarded by imaging of the hepatobiliary system using abdominal ultrasonography.

Biochemical, serological, virological and histological assessments of the patients Biochemical and serological tests:

HBsAg, HBeAg and HBV DNA tests were done at Department of Virology, BSMMU and at LSH. Serum ALT levels and prothrombin time were assessed at Department of Biochemistry, BSMMU. The cut-off value for abnormal ALT was $42 \mathrm{U} / \mathrm{L}$. $\mathrm{HBeAg}$ was checked by ELISA using a kit (Abbott Labs, Chicago, IL, USA). Serum HBV DNA was quantified in a polymerase chain reaction method using a kit (Amplicon HBV Monitor Assay, RTPCR, Roche Molecular Systems, CA, USA). The lower limit of detection was 500 copies of $\mathrm{HBV}$ $\mathrm{DNA} / \mathrm{mL}$.

A percutaneous liver biopsy was performed with written-consent of the patients. Biopsies were performed under local anesthesia using a 16G Trucut biopsy needle (Cardinal Health, McGaw Park, IL, USA). A biopsy specimen of more than $1.0 \mathrm{~cm}$ in length with five to six portal tracts was collected. Histology was graded at Department of Pathology, BSMMU, according to the histologic activity index (HAI) using to the criteria of Knodell et $\mathrm{al}^{\mathbf{1 4}}$. The total HAI score comprises necroinflammation (HAI-NI) and fibrosis (HAI-F) scores. The HAI-NI scale includes three components $(0-10$, piecemeal necrosis; 0-4, lobular necrosis and inflammation; $0-4$, portal inflammation). HAI-F was graded according to severity: 0, absence of fibrosis; 1 , fibrous portal expansion; 3 , bridging fibrosis; 4 , cirrhosis.

\section{Results}

All patients were negative for serological markers of $\mathrm{HAV}, \mathrm{HCV}$ and $\mathrm{HEV}$. In addition, HBsAg was not detected in any of these patients. HBsAg was estimated in 2 different laboratories and negative outcome came from all laboratories. HBV DNA was detected in the sera of 11 of 40 subjects. HBV DNA positivity was confirmed in these patients by checking HBV DNA for at least 3 times in samples taken at different intervals. Also, HBV DNA was checked in two different laboratories. The patients were between 16-57 years of age. Of them, 8 were males and the rest 3 were females. Eight of these patients were diagnosed as liver cirrhosis, 1 had HCC and 2 were apparently healthy individuals with elevated ALT.

Seven of 8 patients with liver cirrhosis exhibited features of hepatic decompensation ( 2 of them had jaundice, 2 had abdominal ascites and 3 had both ascites and jaundine) (Table I). The levels of HBV DNA varied among patients with liver cirrhosis (970 copies/ml to 11000 copies/ml) (Table I). All patients were negative for $\mathrm{HBeAg}$ in the sera. All of them had elevated ALT in the sera (47-213 IU/L). The levels of serum albumin were also low in these patients (16-26 gm/L). Oesophagogastrodudenoscopy revealed grade I varices in 3 patients and portal hypertensive gastropathy in 1 patient (Table I).

The level of HBV DNA was 4300 copies/ml in patient with HCC (Table II). The patient had grade II oesophageal varix on oesophagogastrodudenoscopy. The patient also had liver 
cirrhosis with moderate ascites. A space occupying lesion measuring $5 \times 4.5 \mathrm{~cm}$ was seen in right lobe of liver (Table II).

As shown in Table III, 2 apparently healthy subjects with marginally elevated ALT had low levels of HBV DNA in the sera (590 copies and 680 copies $/ \mathrm{mL}$ ). Liver biopsy revealed mild necroinflammation and minimum hepatic fibrosis in these patients. All HBV DNA-positive patients were expressing anti-HBc in the sera.

Table I: Clinical and laboratory data of eight patients with liver cirrhosis with occult HBV infection

\begin{tabular}{ll}
\hline No. of patients & 8 \\
\hline Age (years) & $16-53$ \\
Male: Female & $5: 3$ \\
Stigmata of chronic liver diseases & 6 \\
Serum ALT $(<42 \mathrm{U} / \mathrm{L})^{*}$ & $47-213 \mathrm{IU} / \mathrm{L}$ \\
HBeAg & negative \\
HBV DNA & $9.7 \times 10^{2}-1.1 \times 10^{4}$ \\
& copies $/ \mathrm{ml}$ \\
Jaundice & 2 \\
Ascites & 2 \\
Jaundice and ascites & 3 \\
Ultrasonographic evidences of liver cirrhosis & 8 \\
Endoscopy & \\
Grade I esophageal varix & 3 \\
Portal hypertensive gastropathy & 1 \\
\hline
\end{tabular}

Profiles of patients with occult HBV infection in patients with liver cirrhosis. *Figure in the parenthesis indicates normal values.

Table II: Clinical and laboratory data of 1 patient with hepatocellular carcinoma with occult HBV infection

\begin{tabular}{ll}
\hline Age & Give the numbers year. \\
\hline Sex & Male \\
Serum bilirubin & $64 \mu \mathrm{mol} / \mathrm{L}$ \\
Serum ALT $(<42 \mathrm{U} / \mathrm{L})$ & $84 \mathrm{IU} / \mathrm{L}$ \\
HBeAg & negative \\
HBV DNA & $4.3 \times 10^{4}$ copies $/ \mathrm{ml}$ \\
Alpha feto protein & $5400 \mathrm{ng} / \mathrm{ml}$ \\
Jaundice & present \\
Ascites & present \\
Features of liver cirrhosis & present \\
Endoscopy & Grade I oesophageal varix \\
\hline
\end{tabular}

Profiles of patient with hepatocellular carcinoma.

Table III: Biochemical, virological and histological assessment of 2 asymptomatic HBV carriers with occult HBV infection

\begin{tabular}{lll}
\hline Parameters & Case 1 & Case 2 \\
\hline Age & 28 yrs. & 41 yrs. \\
Sex & male & male \\
Serum bilirubin & $12 \mu \mathrm{mol} / \mathrm{L}$ & $19 \mu \mathrm{mol} / \mathrm{L}$ \\
Serum ALT $(<42 \mathrm{U} / \mathrm{L})$ & $43 \mathrm{IU} / \mathrm{L}$ & $52 \mathrm{IU} / \mathrm{L}$ \\
HBeAg & negative & negative \\
HBV DNA & $5.9 \times 10^{2}$ copies $/ \mathrm{ml}$ & $6.8 \times 10^{2}$ copies $/ \mathrm{ml}$ \\
Abdominal & normal & normal \\
ultrasonographic & & \\
Endoscopy & normal & normal \\
HAI-NI & 3 & 5 \\
HAI-F & 1 & 1 \\
\hline
\end{tabular}

Profiles of patients with occult HBV infection in asymptomatic HBV-infected subjects. The extent of liver damages was estimated by examining liver biopsy specimens and the levels of necroinflamation and hepatic fibrosis were shown according to the scores of Knodell et al.

\section{Discussion}

Bangladesh is a developing country with a population of 140 million. Like most developing countries of Asia, significant developments have been accomplished for containment of various infectious diseases during last 3 decades. After the eradication of small pox from Bangladesh in late 1970, immunization of children with various vaccines have been started under expanded program of immunization. This has reduced incidence and prevalence of different infectious diseases, such as diphtheria, tetanus, and whopping cough. Children in Bangladesh are also immunized by hepatitis $B(\mathrm{HB})$ vaccine; however, the impact of $\mathrm{HB}$ vaccination on overall $\mathrm{HBV}$ carrier rate has not been adequately assessed. Studies have shown that about 5-8\% apparently healthy people of Bangladesh are chronically infected with the $\mathrm{HBV}^{13}$. Even more alarming is the fact that HBV seems to be major etiological agent of both acute and fulminant hepatitis in Bangladesh. Sugitani et al. have reported that HBV was the main etiological factor of sporadic acute hepatitis, and 36\% adult patients with sporadic acute hepatitis $\mathrm{B}$ in their cohort were infected with the $\mathrm{HBV}^{\mathbf{1 5}}$. Another study conducted by us also revealed that about onethird patients with fulminant hepatic failure were infected with the HBV in Bangladesh ${ }^{\mathbf{1 6}}$. We also found that considerable numbers of these patients became sick after blood transfusion (personal communication) that indicated that transfused blood may have been infected with hepatitis viruses. These facts led us to undertake the present study about entity and prevalence of occult hepatitis B or $\mathrm{HBs} A g-$ negative HBV-infection in Bangladesh.

The public health importance of HBsAg-negative HBV DNA-positive HBV-infected subjects is immense in developing countries like Bangladesh. We detected HBV DNA in 2 of 10 apparently healthy subjects with increased ALT levels. This study reveals that blood donors should be tested for ALT and other markers of HBV, and if possible HBV DNA in Bangladesh. In addition, all patients with liver cirrhosis and HCC should be tested for HBV DNA because anticancer drugs may cause flare of HBV in these patients, and may lead to development of HBV-related fulminant hepatitis $^{\mathbf{1 0 - 1 2}}$. Also, HBV DNA should be checked in patients of different diseases prior to prescribing immune suppressor drugs ${ }^{\mathbf{1 0 - 1 2}}$. Even if HBV DNA can not be estimated for economical and technical reason, anti-HBc should be measured to develop insights if they were infected with HBV. Also, more studies should be performed with $\mathrm{HBsAg}$ negative patients with liver diseases or subjects with elevated ALT. Also, more studies should be performed with HBsAg-negative patients with liver 
diseases or subjects with elevated ALT to assess the impact of these patients on public health.

In conclusion, this study has exposed that $\mathrm{HBsAg}$ should not be regarded as sole marker of HBV infection in developing countries and some mechanisms should be developed to detect HBsAgnegative HBV-infected subjects for containment of HBV infection in developing countries like Bangladesh.

Declaration of interest: There is no conflict of interest regarding this manuscript. We did not receive any fund from for accomplishment of this study. None of the authors have any relation to pharmaceutical company.

\section{References}

1. Ganem D, Prince AM. Hepatitis B virus infectionnatural history and clinical consequences. $\mathrm{N}$ Engl $\mathrm{J}$ Med 2004; 350: 1118-29.

2. Fattovich G, Bortolotti F, Donato F. Natural history of chronic hepatitis B: special emphasis on disease progression and prognostic factors. J Hepatol 2008; 48: $335-52$

3. Liang TJ. Hepatitis B: the virus and the disease. Hepatology 2009; 49: S13-S21.

4. Tsui JI, French AL, Seaberg EC et al. Prevalence and long-term effects of occult hepatitis B virus infection in HIV-infected women. Clin Infect Dis 2007; 45:736-40.

5. Sagnelli E, Imparato M, Coppola N et al. Diagnosis and clinical impact of occult hepatitis B infection in patients with biopsy proven chronic hepatitis C: a multicenter study. J Med Virol. 2008; 80: 1547-53.

6. Bréchot C, Thiers V, Kremsdorf D, Nalpas B, Pol S, Paterlini-Bréchot P. Persistent hepatitis B virus infection in subjects without hepatitis B surface antigen: clinically significant or purely "occult"? Hepatology. 2001; 34: 194-203.
7. Yuan Q, Ou SH, Chen $\mathrm{CR}$ et al. Molecular Characteristics of Occult Hepatitis B virus from blood donors in the southeast China. J Clin Microbiol. 2010; 48: 357-62.

8. Chemin I, Trépo C. Clinical impact of occult HBV infections. J Clin Virol. 2005; 34 Suppl 1:S15-21.

9. Raimondo G, Pollicino T, Squadrito G. What is the clinical impact of occult hepatitis B virus infection? Lancet. 2005 Feb 19-25; 365: 638-40

10. Lok AS, Liang RH, Chiu EK et al. Reactivation of hepatitis B virus replication in patients receiving cytotoxic therapy. Report of a prospective study. Gastroenterology 1991; 100: 182-8.

11. Hui CK, Cheung WW, Zhang HY et al. Kinetics and risk of de novo hepatitis $\mathrm{B}$ infection in $\mathrm{HBsAg}$ negative patients undergoing cytotoxic chemotherapy. Gastroenterology. 2006;131:59-68.

12. Mindikoglu AL, Regev A, Schiff ER. Hepatitis B virus reactivation after cytotoxic chemotherapy: the disease and its prevention. Clin Gastroenterol Hepatol. 2006; 4: 1076-81.

13. Mahtab MA, Rahman S, Karim MF et al Epidemiology of hepatitis B virus in Bangladeshi general population. Hepatobiliary Pancreat Dis Int. 2008; 7:595-600.

14. Knodell RG, Ishak KG, Black WC et al. Formulation and application of a numerical scoring system for assessing histological activity in asymptomatic chronic active hepatitis. Hepatology 1981; 1: 431-5.

15. Sugitani M, Sheikh A, Suzuki K et al. Seroepidemiology of sporadic acute hepatitis in Bangladesh: high prevalences of infection with type-B, type-E and multiple types of hepatitis virus. Ann Trop Med Parasitol. 2009; 103: 343-50.

16. Mahtab MA, Rahman S, Khan M, Mamun AA, Afroz S. Etiology of fulminant hepatic failure: experience from a tertiary hospital in Bangladesh. Hepatobiliary Pancreat Dis Int. 2008; 7: 161-4. 